\title{
Study on Doppler characteristics of underwater bistatic reverberation
}

\author{
Fangyong Wang ${ }^{1, *}$, Yeming Jiang ${ }^{2}$, and Shuanping $\mathrm{Du}^{1}$ \\ ${ }^{1}$ Science and Technology on Sonar Laboratory, 310023 Hangzhou, China \\ ${ }^{2}$ Hangzhou Applied Acoustics Research Institute, 310023 Hangzhou, China
}

\begin{abstract}
Active sonar has two typical types of geometry configuration respectively named monostatic and bistatic like a radar system. In the monostatic scenario, co-located underwater transducers transmit and receive sound energy, while in a bistatic situation the transducers are physically separated. Both object detection and identification can be significantly enhanced through utilization of the additional dimension provided by a bistatic geometry. So, more people care about bistatic scattering characteristics of underwater objects as well as bistatic reverberation in recent years. In this paper, Doppler characteristics of bistatic reverberation generated by moving transmitter and receiver are studied. Theoretical formulism for receiving frequency of bistatic reverberation is derived in case of a tone signal being transmitted. Further analysis shows bistatic reverberation is more complicated than monostatic reverberation when Doppler is concerned. In monostatic case, the Doppler frequency shift of reverberation relates to centre frequency of the tone signal, velocity of the transmitter as well as the arriving direction. While in bistatic situation, it varies not only with the above factors, but also the locations, moving directions, velocities of both transmitter and receiver, and also the arriving time of the reverberation, which makes extraction and utilization of Doppler information more difficult.
\end{abstract}

\section{Introduction}

Active sonar has two typical types of geometry configuration respectively named monostatic and bistatic like a radar system[1]. The distinction originates in the signal transmission and reception configuration. In the monostatic scenario, co-located underwater transducers transmit and receive sound energy, while in a bistatic situation the transducers are physically separated. Although most research and practical application have been in the monostatic case, the bistatic condition offers several key benefits. Both object detection and identification can be significantly enhanced through utilization of the additional dimension provided by a bistatic geometry. The location of the receiver can also keep concealed to the underwater targets to be detected like an attacking submarine. So, more people care about bistatic scattering characteristics of underwater objects as well as bistatic reverberation in recent years[2-4].

Handling and utilization of space and time characteristics of reverberation is important to performance improvement of underwater objects detection. In this paper, we study the way how the receiving frequency of bistatic reverberation vary with time and space by theoretical analysis and computer simulation. The results show that, in bistatic situation, frequency characteristics of underwater reverberation are much more complicated when Doppler is concerned. Specifically, in monostatic case, the Doppler frequency shift of reverberation relates to centre frequency of the tone signal, velocity of the transmitter as well as its direction of arriving. While in bistatic situation, it varies not only with the above factors, but also the locations, moving directions, velocities of both transmitter and receiver, and also the arriving time of the reverberation, which makes extraction and utilization of Doppler information more difficult. Work of this paper may be useful in some sense to bistatic sonar design like reverberation mitigation or reverberation classification. The paper is organized as four parts. The following will include formulation, simulation and a short summary.

\section{Formulations}

\subsection{Geometry of bistatic scattering}

A typical geometry configuration of a bistatic detection system is shown as fig.1. Point $\mathbf{S}$ stands for the transmitting site and $\mathbf{R}$ stands for the receiving site. $\mathbf{T}$ stands for the target to be detected. Relative parameters are denoted as following.

$$
\begin{aligned}
& v_{s}: \text { velocity of transmitting site } \\
& \phi_{s t}: \text { angle from bow of transmitting site to S-T } \\
& \beta_{t}: \text { azimuth angle of target relative to the }
\end{aligned}
$$

transmitting site

Corresponding author: sklwfy@yahoo.com 
$v_{r}$ : velocity of receiving site

$\phi_{r t}$ : azimuth angle of target relative to the receiving site

$f_{0}$ : centre frequency of transmitting signal

$f_{r m}$ : receiving frequency of monostatic echo

$f_{r b}$ : receiving frequency of bistatic echo

$\beta_{b}$ : bistatic angle

$v_{t}$ : velocity of the target

$\theta_{t}$ : course angle of the target

Fig. 1. Geometry configuration of bistatic detection.

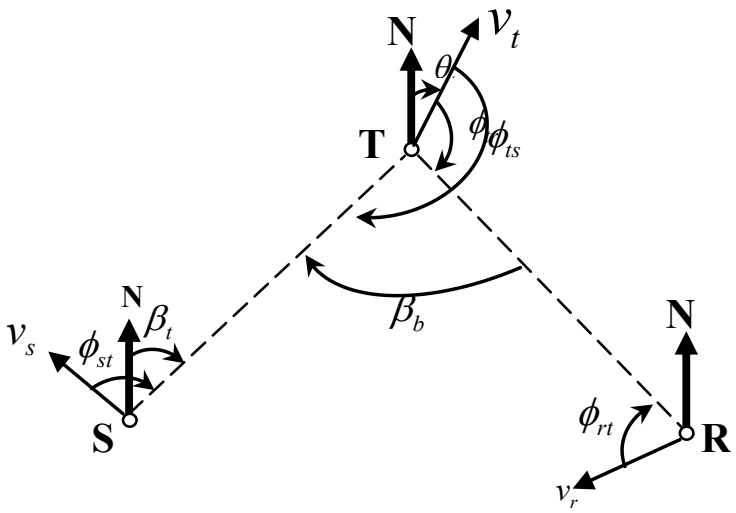

\subsection{The Doppler formulism of bistatic scattering}

In situation of bistatic detection, the transmitting site could also receive echoes which are monostatic. Receiving frequency of these monostatic echoes satisfies

$$
\frac{f_{r m}}{f_{0}}=\frac{c+v_{s} \cos \phi_{s t}}{c-v_{s} \cos \phi_{s t}} \times \frac{c+v_{t} \cos \phi_{t s}}{c-v_{t} \cos \phi_{t s}}
$$

where

$$
\phi_{t s}=\pi-\theta_{t}+\beta_{t}
$$

While the bistatic echoes received by receiving site will satisfy

$$
\frac{f_{r b}}{f_{0}}=\frac{1}{c-v_{s} \cos \phi_{s t}} \times \frac{c+v_{t} \cos \phi_{t s}}{c-v_{t} \cos \phi_{t r}} \times\left(c+v_{r} \cos \phi_{r t}\right)
$$

where,

$$
\phi_{t r}=\pi-\theta_{t}+\beta_{t}-\beta_{b}
$$

By one-order approximation, that is

$$
\begin{gathered}
\frac{f_{r m}}{f_{0}}=1+2 \frac{v_{s}}{c} \cos \phi_{s t}+2 \frac{v_{t}}{c} \cos \left(\pi-\theta_{t}+\beta_{t}\right) \\
\frac{f_{r b}}{f_{0}}=1+\frac{v_{s}}{c} \cos \phi_{s t}+\frac{v_{t}}{c} \cos \left(\pi-\theta_{t}+\beta_{t}\right)+ \\
\frac{v_{t}}{c} \cos \left(\pi-\theta_{t}+\beta_{t}-\beta_{b}\right)+\frac{v_{r}}{c} \cos \phi_{r t}
\end{gathered}
$$

Above equations describe the relation between receiving frequency of monostatic or bistatic echoes and other measured parameters.
For bistatic echoes, the Doppler frequency shift caused by target moving will be

$$
\begin{aligned}
f_{e} & =\frac{c+v_{t} \cos \phi_{t s}}{c-v_{t} \cos \phi_{t r}} \approx 1+\frac{2 v_{t}}{c}\left(\frac{\cos \phi_{t s}+\cos \phi_{t r}}{2}\right) \\
& =1+\frac{2 v_{t}}{c} \cos \theta_{e}
\end{aligned}
$$

We can easily get from above equation that, in bistatic case, doppler shift caused by target moving is determined not only by the moving speed of the target, but also by the two angles $\phi_{t r}$ and $\phi_{t s}$, which are jointly generated by locations of the target, transmitting site and receiving site.

According to equation (2), receiving frequency of echoes reflected by motionless clutters will be

$$
f_{r e v b}=\frac{c+v_{r} \cos \phi_{r t}}{c-v_{s} \cos \phi_{s t}} f_{0}
$$

which means frequency of clutter-caused reverberation received by receiving site will be related to speed of the two sites and angle pairs $\left(\phi_{r t}, \phi_{s t}\right)$ which are denoted in fig.1. So, the frequency will not be a cosine function of arriving angle as in monostatic situation. And also, the frequency of reverberation will be time or distance dependent.

\section{Simulations}

According to above analysis, we have studied the frequency characteristics of reverberation with different arriving angles and distances as following.

(1) Locations and course angles of transmitting site and receiving site are shown as Fig.2. The two sites both run with speed of $14 \mathrm{knots}$. The course angle of receiving site keeps 0 degree. Distance between the two sites is $14 \mathrm{~km}$. The centre frequency of the transmitted signal is $1825 \mathrm{~Hz}$ and this value will keep the same in following simulation. Fig. 3 shows the varying of receiving frequency with arriving angle and distance. Every subfigure corresponds to different course angles of transmitting site. Fig.4 shows the distance-frequency curve of reverberation at some typical distances of arriving angles.

Fig.3-4 show that, in bistatic case, the frequency of reverberation relates not only with the arriving angle as the monostatic case, but also with the arriving distance( or time). And the pattern is determined by the geometry configuration of the two sites.

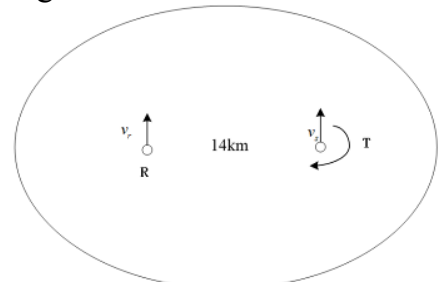

Fig. 2. Geometry configuration of transmitting site and receiving site. 

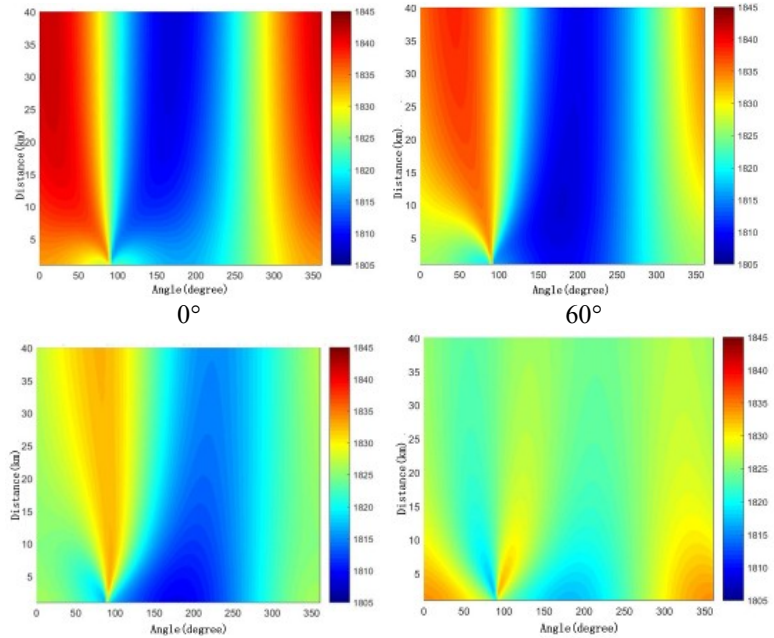

$120^{\circ}$

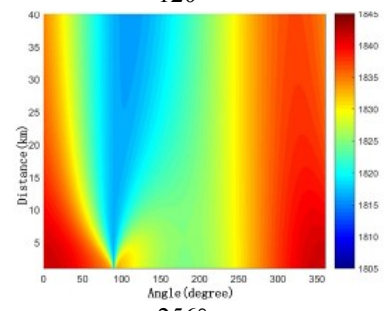

$256^{\circ}$
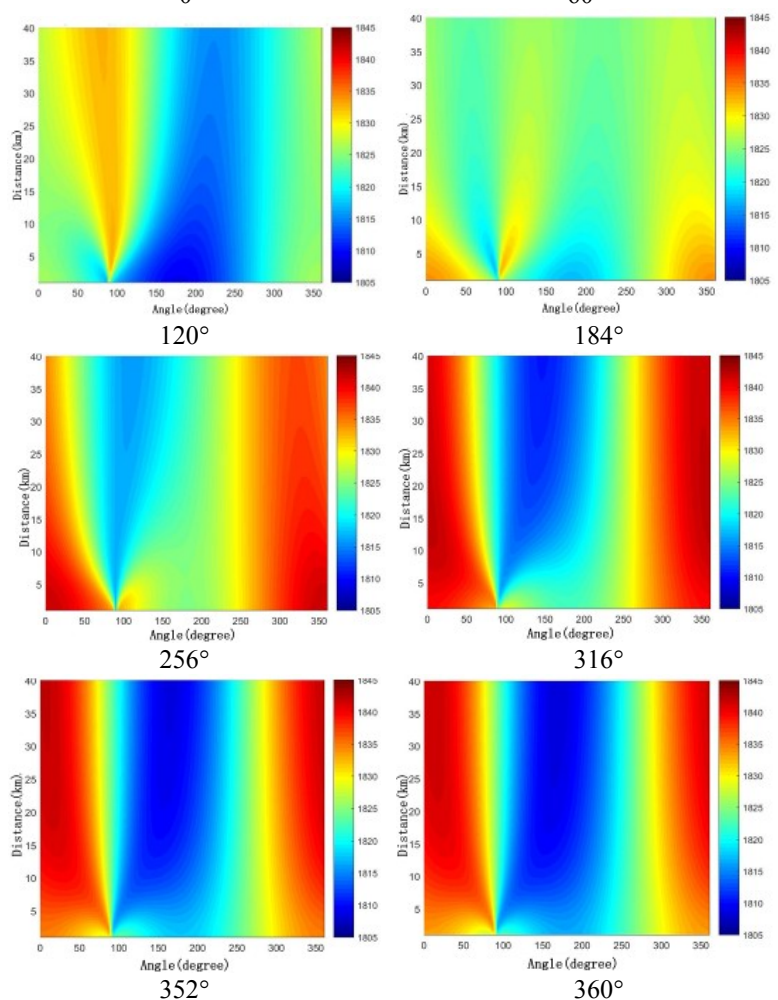

$184^{\circ}$

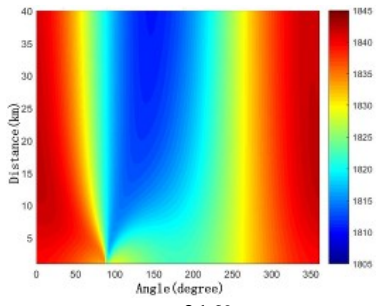

$316^{\circ}$

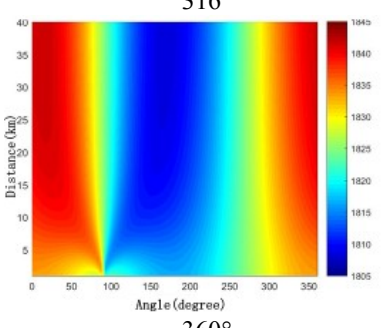

$360^{\circ}$

Fig. 3. Angle-distance spectrum of reverberation at different course angles of transmitting site.
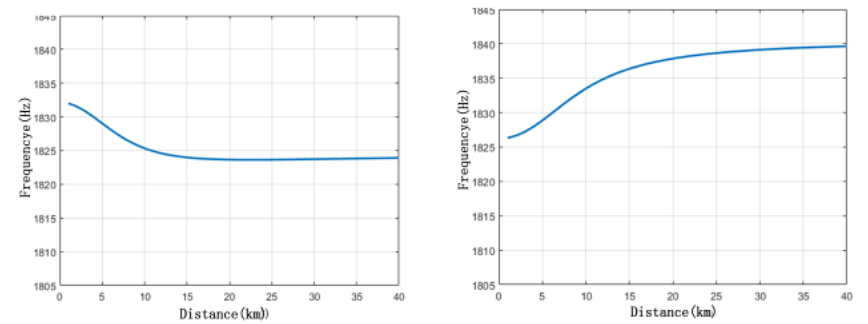

Fig. 4. Distance-frequency curve of reverberation at arriving angles of 45 degrees for both left side(left) and right side(right).

(2) Locations and course angle of transmitting site and receiving site are shown as Fig.4. The two sites both run with speed of $14 \mathrm{knt}$. Course angles of the two sites are 270 and 90degrees respectively. Fig.5 shows the different angle-distance spectrum of reverberations when distance between the transmitting site and receiving site changes from 0.2 to $20 \mathrm{~km}$.

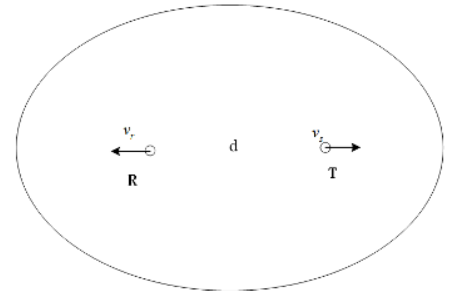

Fig. 5. Geometry configuration of transmitting site and receiving site.
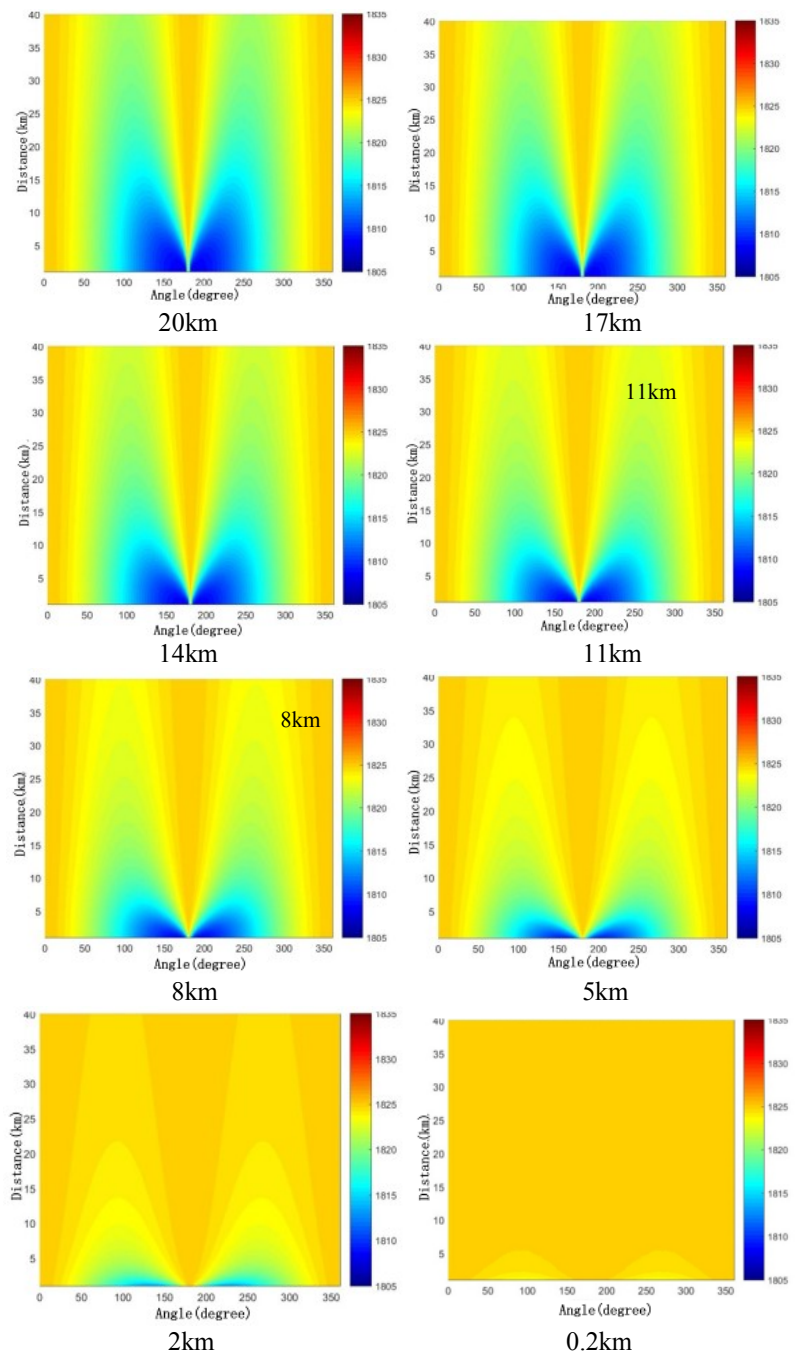

Fig. 6. Angle-distance spectrum at different transmit-receive distance.

It is shown in fig.6 that, when $V_{r}=-V_{s}$ and the two sites near enough to each other, the frequency characteristics of reverberation will almost not change with distance. And the whole bistatic system equalises to a monostatic system with no speed.

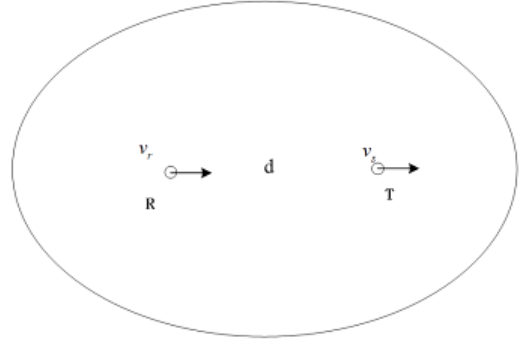

Fig. 7. Geometry configuration of transmitting site and receiving site.

(3) Locations and course angle of transmitting site and receiving site are shown as Fig.7. The two sites both run with speed of $14 \mathrm{knt}$. The course angle of the two sites is both 90 degrees. Distance between the two sites is $14 \mathrm{~km}$. Fig. 5 shows the different angle-distance spectrum of reverberations when distance between the transmitting site and receiving site changes from $0.2 \mathrm{~km}$ to $20 \mathrm{~km}$. 


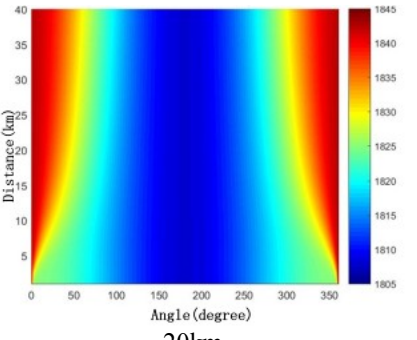

$20 \mathrm{~km}$

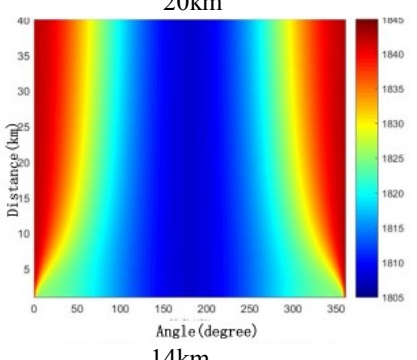

$14 \mathrm{~km}$

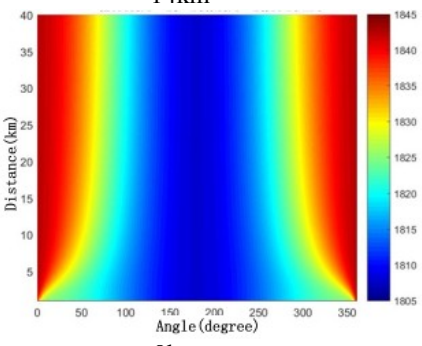

$8 \mathrm{~km}$

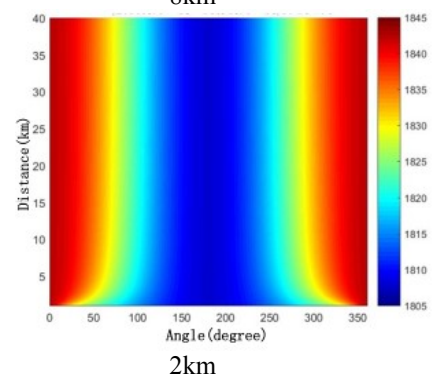

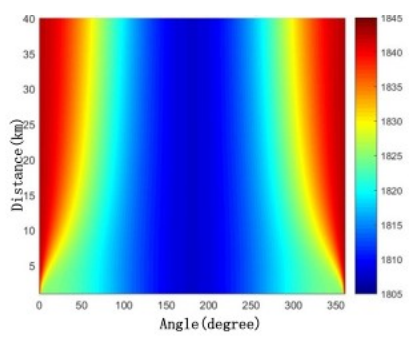

$17 \mathrm{~km}$

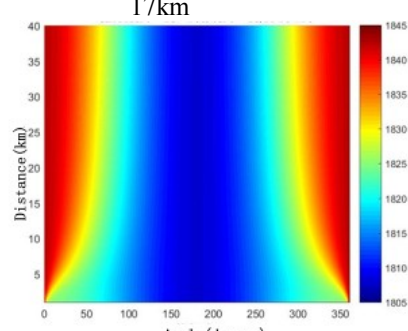

$11 \mathrm{~km}$

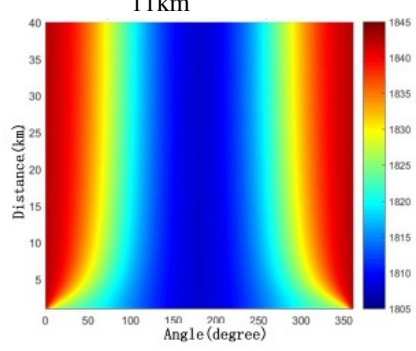

$5 \mathrm{~km}$

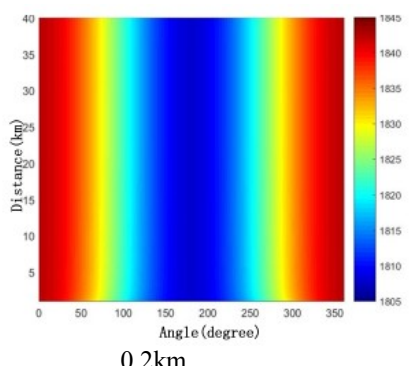

$0.2 \mathrm{~km}$

Fig. 8. Angle-distance spectrum with different transmit-receive distance.

It is shown in Fig.8 that, when $V_{r}=V_{s}$ and the two sites near enough to each other, the frequency characteristics of reverberation will almost not change with distance, and the whole bistatic system equalises to a monostatic system moving with speed of $V_{r}$.

\section{Conclusions}

In this paper, Doppler characteristics of bistatic reverberation generated by moving transmitter and receiver are studied. Theoretical formulism for receiving frequency of bistatic reverberation is derived in case of a tone signal being transmitted. Further analysis shows bistatic reverberation is more complicated than monostatic reverberation when Doppler is concerned. Range or time dependence is the main difference between the two cases. In monostatic case, the Doppler frequency shift of reverberation relates to centre frequency of the tone signal, velocity of the transmitter as well as the arriving direction. It has nothing to do with arriving time or range. While in bistatic situation, it varies not only with the above factors, but also the

locations, moving directions, velocities of both transmitter and receiver, and also the arriving time or range of the reverberation, which makes extraction and utilization of Doppler information more difficult. And actually, the latter is just a special case of the former.

\section{References}

1. C.Henry, Fundamentals of bistatic active sonar, in NATO Underwater Acoustic Data Processing, Kingston, ON, Canada, Jul. (1988).

2. M. Vertner Brown R. Alfred Saenger, J. Acoust. Soc.52, 3(1972)

3. Pascal A. M. de Theije and Jean-Christophe Sindt, IEEE. JOE, 31, 1(2006).

4. John R. Preston and Wayne A. Kinney, J. Acoust. Soc. Am. 93 (5), May 1993 